\title{
Investigating the Impact of Random Waypoint AND Vector Mobility Models on AODV, OLSR and GRP Routing Protocols in MANET
}

\author{
Sohajdeep Singh \\ M.Tech. Computer Science \\ Research Scholar \\ DAVIET Jalandhar
}

\author{
Parveen Kakkar \\ Assistant Professor \\ 1st Department of CSEDAVIET Jalandhar
}

\begin{abstract}
A Mobile Ad hoc Network (MANET) is a collection of wireless mobile nodes forming a temporary network without the need for base stations or any other preexistingnetwork infrastructure.Due to link instability, node mobility and frequently changing topologies routing becomes one of the core issues in MANETs.this paper examine Random Waypoint Mobility model and Vector Mobility model and study their impact on AODV, OLSR and GRP routing protocols with Throughput ,End-to-End Delay and Network Load as Performance Metrics.
\end{abstract}

\section{Keywords}

MANET, AODV, OLSR, GRP, Random Waypoint Mobility Model, Vector Mobility model.

\section{INTRODUCTION}

With the advent of new technologies such as Bluetooth and Hyperlan, the possibility of implementation of MANETs[16] have extended vastly and it can nowadays be implemented in a commercial level on a broad range of applications such as distributed mobile computing (e.g., sensors, disaster relief ) war front activities and communication among automobiles on highways. through enlargement of its scope of implementation, the challenge is to formulate efficient routing protocols which are capable to handle high degree of mobility and randomness in movement of nodes. Furthermore, each node which participates in a MANET plays the double role of a terminal and a router, while a central controlling authority is absent. Conventional routing protocols which are used in fixed networks be unsuccessful in doing so. The reliability and performance of MANETs can be improved by using algorithms which are especially tailored for such networks.Many routing protocols[16][23] have beendeveloped by using various routing mobility metrics to choose the most reliableroutes, while dealing with the primary obstacle caused by node mobility. The objective of this paper is to examine Random Waypoint Mobility model and Vector Mobility model and study their impact on AODV, OLSR and GRP routing protocols with Throughput ,End-to-End Delay and Network Load as Performance Metrics.'

\subsection{Routing Protocols}

There are various protocols already have developed for MANET environment. All these protocols can be classified in diverse ways. Based on the network structure the routing protocols can be classified as Proactive, Reactive and Hybrid routing.

\subsubsection{Proactive Routing Protocols}

Proactive or table-driven routing protocols preserve fresh lists of destinations and their routes by distributing routing tables in the network periodically. The Proactive routing protocolthat this paper will study is calledOptimized Link State Routing (OLSR)[26].

Optimized Link State Routing Protocol OLSR is a modular proactive hop by hop routing protocol. It provide fresh path of destination for table driven approach. It is an optimization of pure link state algorithm in ad hoc network. The routes are always immediately available when needed due to its proactive nature. The key concept of the protocol is the use of "multipoint relays" (MPR). Each node selects a set of its neighbor nodes as MPR. Only nodes, selected as such MPRs are responsible for generating and forwarding topology information, intended for diffusion into the entire network. The MPR nodes can be selected in the neighbor of source node. Each node in the network keeps a list of MPR nodes. This MPR selector is obtained from HELLO packets sending between in neighbor nodes. These routes are built before any source node intends to send a message to a specified destination In order to exchange the topological information; the Topology Control (TC) message is broadcasted throughout the network.

\subsubsection{Reactive or on-demand routing protocols}

Reactive or on-demand protocols find routes on demand byflooding the network with Route Request packets. This allows

only the routes that the network needs to be entered into a routing table. The reactive protocols will be investigated in this paper is Ad hocOn-demand Distance Vector (AODV)[18][23].

It is an on-demand protocol, thus it reduces the overheads by not requiring routing information till a request for path is generated. The source broadcasts a route request (RREQ) packet when it wants to find path to the destination. The neighbors in turn broadcast the packet to their neighbors until it reaches an intermediate node that has recent route information about the destination or until it reaches the destination. When a node forwards a RREQ to its neighbors, it also records in its tables the node from which the first copy of the request came. This information is used to construct the reverse path for the route reply packet (RREP). AODV requires the maintenance of timer based states in each node regarding utilization of individual routing table entries. 


\subsubsection{Hybrid Routing Protocols}

Hybrid Routing Protocols Combine the advantage ofReactive and Proactive Routing Protocols. In this Paper Gatheringbased routing protocol (GRP) will be investigated.

Gathering-based routing protocol (GRP) combines the advantages of Proactive Routing Protocol (PRP) and of Reactive Routing protocol (RRP). PRP are suitable for supporting the delay sensitive data such as voice and video but it consumes a great portion of the network capacity, while RRP is not suitable for real-time communication. The function of Gathering-based Routing Protocol (GRP) for mobile ad hoc network is to gather network information rapidly at a source node without spending a large amount of overheads. It offers an efficient framework that can simultaneously draw on the strengths of Proactive routing protocol (PRP) and reactive routing protocol (RRP) collects network information at a source node at an expense of a small amount of control overheads.

\subsection{MOBILITY MODELS}

In MANETs, mobile nodes move around in the network topology whichmake it hard to model the definite pattern of node mobility in a actual life mobility[1][15] pattern.therefore, approximation of movement pattern is resorted to. Mobility models describe the movements of mobile nodesby providing for variation in speed and direction in regular time intervals. Some of the mobility models in MANETs are as follows:

- Random way point mobility model

- Random walk mobility model

- Reference Point Group Mobility model

- Vector mobility model

- Manhattan Mobility Model

- Chain mobility model

In this paper we examine Random Waypoint Mobility model and Vector Mobility model and study their impact on AODV, OLSR and GRP routing protocols. The Random Waypoint Mobility model and Vector Mobility model have been discussed as follows:

1.2.1 Random Waypoint Mobility model:In this model, the position of each node is randomly selected within a fixed area and after that moves to the selected position in linear form with random speed. This movement has to stop by a certain period called pause time before starting the next movement.

1.2.2 Vector Mobility model:This model is used to avoid the impractical behaviour which is actually impossible. By remembering mobility state of a node and allow only partial change in the current mobility state, natural motions can be reproduced.

\section{LITERATURE SURVEY}

Juan-Carlos Cano et.al[5] in their paper "Group mobility impact over TCP and CBR traffic in Mobile Ad Hoc Networks"(2004).This paper presents an analysis of the effect that mobility models have over the performance of a mobile ad hoc network. Using CBR, it is observed that the general the end-to-end delay increases as the hops count increases. With the random waypoint model the delay can be almost three times higher with respect to group mobility models. They make evident that group mobility pattern highly affects the performance of both CBR traffic and TCP traffic

In Bai, Fan[15], et.al, "A Survey of Mobility Models in Wireless Adhoc Networks.", (Chapter 1 in Wireless Ad-Hoc
Networks. Kluwer Academic)(2006),Beside the commonly used Random Waypoint model and its variants, they also discuss various models that exhibit the characteristics of temporal dependency, spatial dependency and geographic constraint. V(maximum allowable velocity) and $\mathrm{T}$ (pause time)are the two key parameters that determine the mobility behavior of nodes for every mobile node.

Liu Tie-yuan[8] et.al in their study "Analyzing the Impact of Entity Mobility Models on the Performance of Routing Protocols in the MANET" (2009) presents a comparative study on entity mobility models. The RWP model has the highest delivery ratio, lowest end-to-end delay, and shortest average hop count, the RD model just reversed, and the RW, MRP model are between these two models.

SabinaBaraković[6] et.al, (2010) "Comparison of MANET Routing Protocols in Different Traffic and Mobility Models"; This paper compares performances of three routing protocols: Destination Sequenced Distance Vector (DSDV), Ad Hoc On demand Distance Vector (AODV) and Dynamic Source Routing (DSR). In low load and low mobility scenarios routing protocols perform in a similar manner. However, with mobility and load increasing DSR outperforms AODV and DSDV protocols.

K. Sreenivasulu[4], et.al, (2011)“Improving Routing Efficiency Based On Random Direction Mobility Model In MANET" They Compare probability and simulator results to obtainroute stability, so as to Improve routing efficiency as well by Random Direction Mobility Model.

DimitriPerrin[1] et.al, (2012) "Impact of Mobility and Topology on Information Diffusion in MANETs".This paperintroduced a modelling framework for computer-based analysis of epidemic broadcasts. In particular, they look at the impact of bias in path selection for a number of network configurations in both regular and irregular network structures. Simulation results showed that bias in agent mobility (towards the shortest path) significantly improves the information dissemination speed.

Anil Bhasin and Dinesh Kumar[3] (2012) "Comparative Study of Chain Mobility Models using Reactive Routing protocols".In this paper they focus on the impact of Chain mobility models on the performance of Reactive routing protocols. On the basis of end-to-end delay, the DSR performs better than AODV. But when number of nodes increase, AODV performs better. On the mobility front, Chain campus performs well in low to medium density.

Shaveta and Krishan Kumar Saraswat[2] (2012)"Pursue Shortest Mobility Model and Its Comparison in MANET" in this paper they proposed the new mobility model called Pursue Shortest Mobility Model and measure the performance with three routing protocols i.e. AODV, OLSR, GRP.

\section{METHODOLOGY USED}

\subsection{Simulation Setup}

We used Network Simulation OPNET (optimized Network Engineering Tool) Modeler version 14.5 in our evaluation. The OPNET is a discrete event driven simulator. It simulates the network graphically and its graphical editors mirror the structure of actual networks and network components. The users can design the network model visually. The modeler uses object-oriented modeling approach. The nodes and protocols are modeled as classes with inheritance and specialization. The development language is $\mathrm{C}$. The simulation is performed to evaluate the performance of routing protocols with the vector mobility and random waypoint mobility issue at HTTP traffic. Therefore, different simulation scenarios consisting of 25 nodes for AODV OLSR and GRP is considered. The nodes 
were randomly placed within certain gap from each other in $3.5 \times 3.5 \mathrm{~km}$ office environment for 25 nodes. The constant HTTP traffic is generated in the network explicitly i.e. user defined via Application configuration and Profile Configuration. Every node in the network was configured to execute AODV, OLST and GRP respectively. The simulation time was set to 5 minutes and all the nodes were configured with defined mobility in space.

\subsection{Simulation Parameters}

Table 1:Simulation Parameters

\begin{tabular}{|c|c|}
\hline Parameter & Value \\
\hline Simulator & Opnet 14.5 \\
\hline Area & $3.5 \times 3.5 \mathrm{Km}$ \\
\hline Wireless MAC & 802.11 \\
\hline Number Of Nodes & 25 \\
\hline Mobility Model & $\begin{array}{c}\text { Vector Mobility,Random } \\
\text { Waypoint Mobility }\end{array}$ \\
\hline Data Rate & 11 Mbps \\
\hline Routing Protocols & AODV,OLSR And GRP \\
\hline Simulation Time & 5 minutes \\
\hline
\end{tabular}

\subsection{PERFORMANCE PARAMETERS}

The following Performance Metrics has been used for evaluating the performance of various MANET routing protocols:

- Network Load: The statistic represents the total data traffic (in bits/sec) received by the entire WLAN BSS from the higher layers of the MACs that is accepted and queued for transmission

- End-to-end Delay: Represents the end to end delay of all the packets received by the wireless LAN MACs of all WLAN nodes in the network and forwarded to the higher layer. This delay includes medium access delay at the source MAC, reception of all the fragments individually, and transfers of the frames via access point, if access point functionality is enabled.

- Throughput: Represents the total number of bits (in bits/sec) forwarded from wireless LAN layers to higher layers in all WLAN nodes of the network.

\section{RESULT ANALYSIS}

The simulation results are shown in this section in the form graphs. Graphs show comparison between the three protocols by varying different numbers of sources on the basis of the above-mentioned metrics:

\subsection{END TO END DELAY}

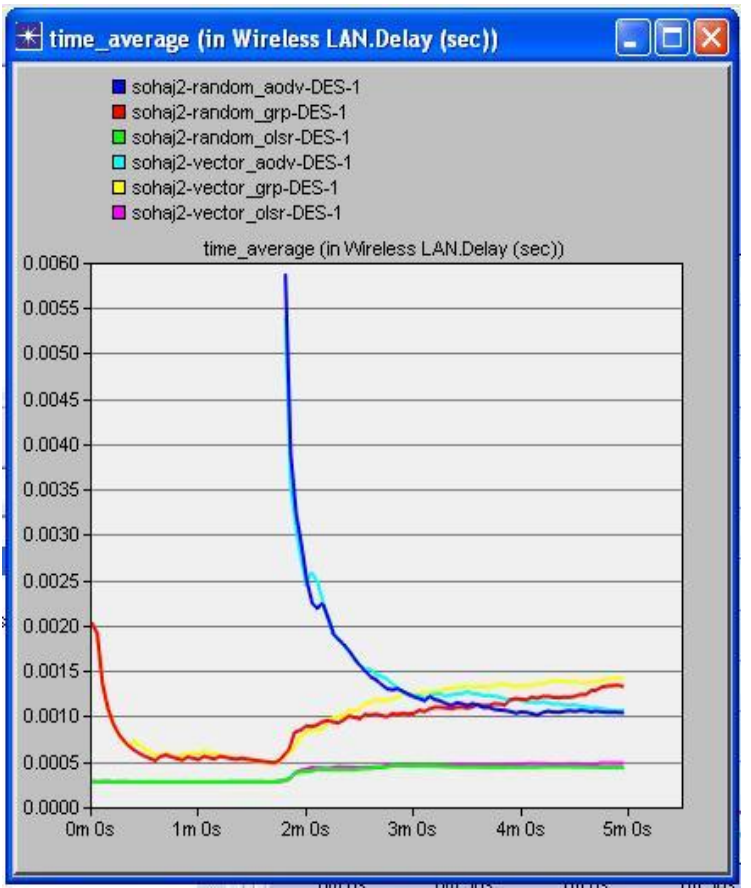

Fig 1: End-to-end Delay

Figure 1 shows the performance of AODV, OLSR and GRP by evaluating End to End Delay with Vector Mobility and random waypoint mobility Model 25 numbers of sources(S) with HTTP traffic. It is the result of the time of data packets delivered to the destination nodes minus the time of data packets transmitted by the source nodes, and then divided by the number of data packets received bydestination nodes.Represents the end to end delay of all the packets received by the wireless LAN MACs of all WLAN nodes in the network.

\subsection{THROUGHPUT}

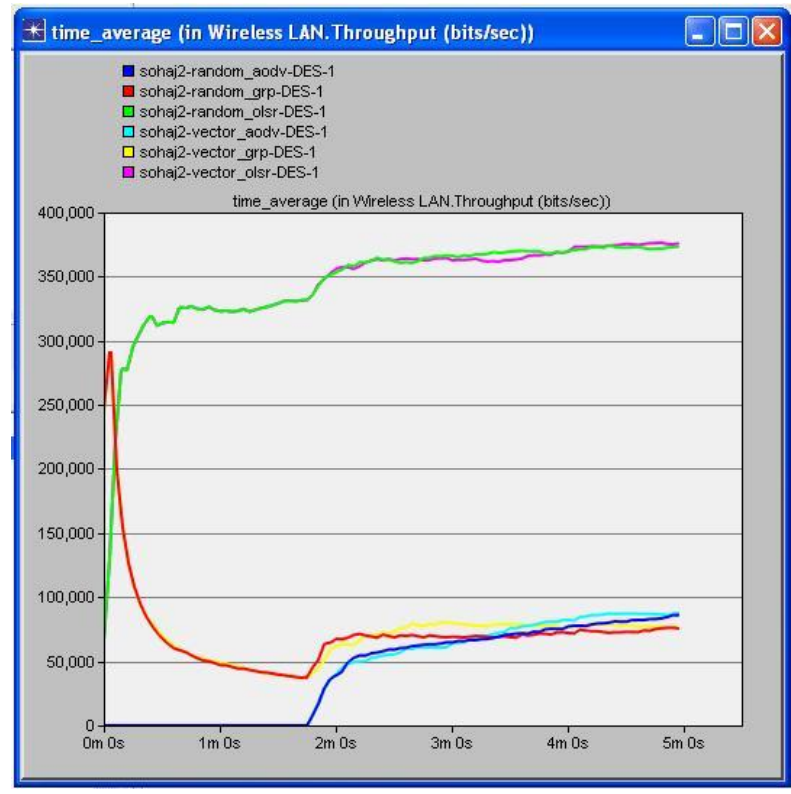

Fig 2: Throughput 
It is the total number of bits (in bits/sec) forwarded from wireless LAN layers to higher layers in all WLAN nodes of the network.The average throughput for the network with 25 nodes is shown in Figure 2 which reflects the usage degree ofthe network resources for the typical routingprotocol.With, the maximum throughput is approximately $45.77 \mathrm{kbps}$. Throughput increases quickly for OLSR with increased number of nodes. While AODV and GRP on the other hand has difficulties in finding routes when number increases, which clear from the figure.Figure 3 shows that OLSR perform better then AODV and GRP routing protocols.

\subsection{NETWORK LOAD}

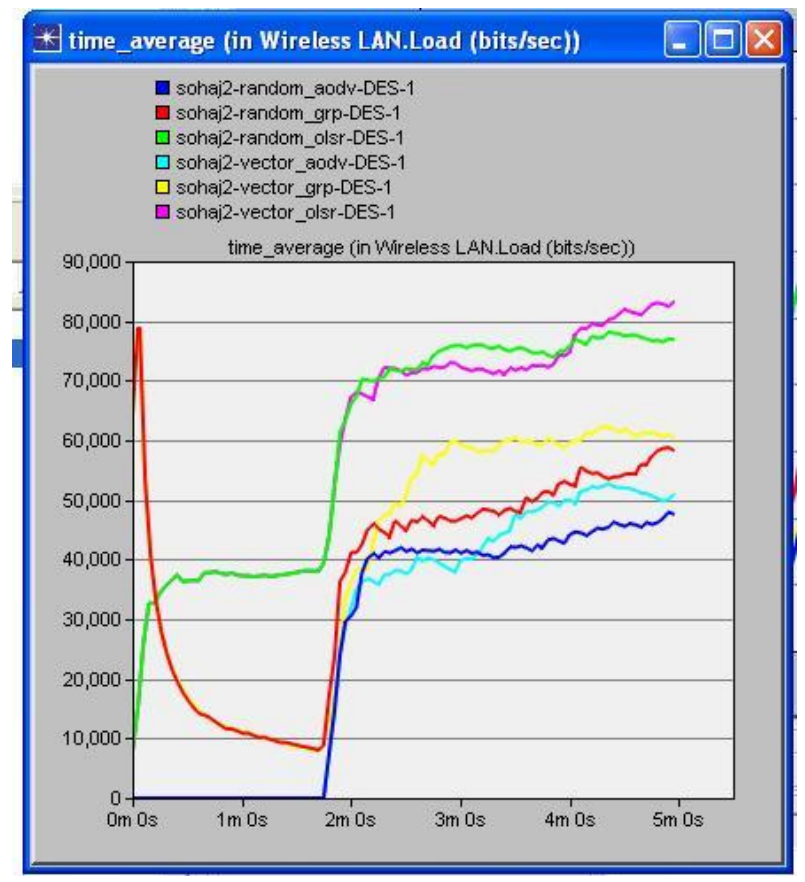

Fig 3: Network Load

Figure 3 shows Network Load. Here network load for Vector Mobility and random waypoint mobility. It is the total data traffic (in bits/sec) received by the entire WLAN Here AODV for both mobility models having lesser network load than others, while OLSR has the highest Network Load in the network.

\section{CONCLUSION AND FUTURE SCOPE}

In this paper we evaluated the three performance measures i.e. Network Load, End-to-end delay and Throughput with different mobility models (Vector Mobility model and and Random Waypoint Mobility model) and HTTP as traffic type. From the extensive simulation results, it is found that OLSR shows the best performance in terms of throughput, and endto-end delay.

In future, the node density can be varied to study its impact on the performance of the routing protocols and thus check their efficiency as the nodes increase. Also, the type of traffic can be varied to types other than the one we use (heavy HTTP). FTP and VoIP are a few suggested traffic types that may be implemented to study the change in the efficiency of our routing protocols. Utilizing these performance outputs, we can design such a protocol that can suitably provide data integrity as well as data delivery in highly random mobilitynetwork.

\section{ACKNOWLEDGMENTS}

My sincere thanks to my guide Er.ParveenKakkar, who provided vital inputs and guidance on important issues faced in the choice and implementation of the problem discussed above. I would also like to thank the D.A.V.I.E.T. for allowing me to use its research facility.

\section{REFERENCES}

[1] Dimitri Perrin, Hiroyuki Ohsaki 2012 Impact of Mobility and Topology on Information Diffusion in MANETs. IEEE.

[2] Shavetaand Krishan Kumar Saraswat2012 Pursue Shortest Mobility Model and Its Comparison in MANET. VSRD International Journal of CS \& IT Vol. 2 (6) pp 462-472

[3] Anil Bhasin , Dinesh Kumar 2012. Comparative Study of Chain Mobility Models using Reactive Routing protocols. International Journal of Computer Applications (0975 - 8887) Volume 42- No.9, March 2012

[4] K. Sreenivasulu1, Mr. A.L. Srinivasulu2011. Improving Routing Efficiency Based On Random Direction Mobility Model In Manets. International Journal of Smart Sensors and Ad Hoc Networks (IJSSAN) Volume1 , Issue-1.

[5] Juan-Carlos Cano and Pietro Manzoni 2011. Group mobility impact over TCP and CBR traffic in Mobile Ad Hoc Networks. IEEE

[6] Sabina Baraković, SuadKasapović, and JasminaBaraković2010. Comparison of MANET Routing Protocols in Different Traffic and Mobility Models.TelforJournal, Vol. 2, No. 1,.

[7] S. R. Biradar, Hiren H D Sharma, Kalpana Sharma, Subir Kumar Sarkar, Puttamadappa C 2009. Performance Comparison of Reactive Routing Protocols of MANETs using Group Mobility Model IEEE.

[8] Liu Tie-yuan, CHANGLiang, GuTian-long 2009 Analyzing the Impact of Entity Mobility Models on the Performance of Routing Protocols in the MANET. IEEE.

[9] Suresh Kumar, R.K. Rathy and DiwakarPandey2009. Traffic Pattern Based Performance Comparison of Two Reactive Routing Protocols for Ad-hoc Networks using NS2. $2^{\text {nd }}$ IEEE International Conference on Computer Science and Information Technology.

[10] Opnet Technologies, Inc. “OPNET Simulator,” Internet: www.opnet.com, date last viewed: 2009-12-21.

[11] S. R. Biradar, Hiren H D Sharma, KalpanaShrama and Subir Kumar Sarkar2009. Performance Comparison of Reactive Routing Protocols of WSNs using Group Mobility Model", IEEE International Conference on Signal Processing Systems, pages 192-195.

[12] N.Aschenbruck, E.Gerhands-Padilla, P.Martini 2008. A Survey on mobility models for Performance analysis in Tactical Mobile networks.Journal of Telecommunication and Information Technology, Vol.2 pp.54-61.

[13] K. Salah, P. Calyam, and M. I. Buhari2007. Assessing Readiness of IP Networks to Support Desktop Video Conferencing Using OPNET.Journal of Network and Computer Applications, no. pp.1-23. 
[14] D. Johnson, Y. Hu, and D. Maltz2007. The Dynamic Source Routing Protocol (DSR) for Mobile”, RFC 4728.

[15] Bai Fan, Helmy, Ahmed 2006. A Survey of Mobility Models in Wireless Adhoc Networks. Chapter 1 in Wireless Ad-Hoc Networks. Kluwer Academic.

[16] 16. L. Hogie, P. Bouvry, and F. Guinand2006. An Overview of MANET Simulation. ElectronicNotes in Theoretical Computer Science, vol. 150, no. 1, pp. 81101.

[17] 17. S.S. Miremadil, M. Fazelil, A. Patooghyl, S.G. Miremadi 2006. Performance Evaluation of a Routing Protocol for Wireless Sensor Networks. IEEE.

[18] Anipakala Suresh 2005. Performance Analysis of Ad hoc On-demand Distance Vector Routing (AODV) Using OPNET Simulator Communication Networks. University of Bremen.

[19] K. Akkaya and M. Younis2005. A survey of Routing Protocols in Wireless Sensor Networks. Elsevier Ad Hoc Network Journal, pp 325-349.

[20] Juan-Carlos Cano, Pietro Manzoni and Miguel Sanchez 2004. Evaluating the impact of group mobility on the performance of mobile ad hoc networks. IEEE.

[21] HasnaaMoustafa and HoudaLabiod2004. A Performance Analysis of Source Routing-based Multicast Protocol (SRMP) Using Different Mobility Models. IEEE.
[22] F. Bai, N Sadagopan, A. Helmy2003. IMPORTANT: A framework to systematically analyze the Impact of Mobility on Performance of Routing Protocols for Adhoc Networks. IEEE INFOCOM.

[23] S. Das, C. E. Perkins, E. Royer 2002. Ad Hoc On Demand Distance Vector (AODV) Routing. IETF Draft.

[24] P. Jacquet, P. Muhlethaler, T. Clausen, A.Laouiti, A. Qayyum, L. Viennot. Optimized Link State Routing Protocol for Ad Hoc Networks. Hypercom Project, INRIA Rocquencourt, BP 105, 78153 Le ChesnayCedex, France.

[25] Jing Xie, Luis Girons Quesada and YumingJiang . A Threshold-based Hybrid Routing Protocol for MANET. Department of Telematics, Norwegian University of Science and Technology.

[26] Ying Ge. Quality of Service Routing in Ad-Hoc Networks Using OLSR" Proceedings of the 36th Hawaii International Conference on System Sciences (HICSS’03)0-7695-1874-5/03 \$17.00 @ 2002 IEEE.

[27] C.E. Perkins and E.M.Royer, "Ad-Hoc On Demand Distance Vector Routing", Proceedings of the 2nd IEEE Workshop on Mobile Computing Systems and Applications, New Orleans, LA, USA, pages 90-100, February 1999.

[28] X. Chang, "Network Simulations with OPNET," Presented at Simulation Conference Proceedings, 1999 Winter, 1999, pp. 307-314. 